\title{
TITLE:
}

\section{High time resolution RPCs with different readout geometries}

\section{$\operatorname{AUTHOR}(\mathrm{S})$ :}

Tomida, N; Hsieh, C-Y; Niiyama, M; Ohnishi, H; Chang, W-C; Hashimoto, T; Hamano, H; Mizutani, K

\section{CITATION:}

Tomida, N ...[et al]. High time resolution RPCs with different readout geometries. Journal of Instrumentation 2012, 7(12): P12005.

\section{ISSUE DATE:}

2012-12-06

URL:

http://hdl.handle.net/2433/173354

\section{RIGHT:}

(c) 2012 IOP Publishing; Open access articles from IOP are published under a creative commons attribution licence or CC-BY Licence (see creative commons definitions below). This allows users to re-use the content in any way they like, provided it is accurately attributed. Copyright stays with the agreed copyright owner. 
High time resolution RPCs with different readout geometries

This article has been downloaded from IOPscience. Please scroll down to see the full text article.

2012 JINST 7 P12005

(http://iopscience.iop.org/1748-0221/7/12/P12005)

View the table of contents for this issue, or go to the journal homepage for more

Download details:

IP Address: 130.54.110.72

The article was downloaded on 18/04/2013 at 03:21

Please note that terms and conditions apply. 


\title{
High time resolution RPCs with different readout geometries
}

\section{N. Tomida ${ }^{a}{ }^{1}$ C.-Y. Hsieh,${ }^{b}$ M. Niiyama, ${ }^{a}$ H. Ohnishi, ${ }^{c}$ W.-C. Chang, ${ }^{b}$ T. Hashimoto, ${ }^{a}$ H. Hamano ${ }^{d}$ and K. Mizutani ${ }^{a}$}

\author{
${ }^{a}$ Department of physics, Kyoto University, \\ Kitashirakawa-Oiwakecho, Sakyo-ku, Kyoto 606-8502, Japan \\ ${ }^{b}$ Academia Sinica, \\ 128 Academia Road, section 2, Nankang, Taipei 115, Taiwan \\ ${ }^{c}$ RIKEN (The Institute of Physical and Chemical Research), Nishina Center, \\ 2-1 Hirosawa, Wako, Saitama 351-0198, Japan \\ ${ }^{d}$ Research Center for Nuclear Physics (RCNP), Osaka University, \\ 10-1 Mihogaoka, Ibaraki, Osaka 567-0047, Japan \\ E-mail: natsuki@scphys.kyoto-u.ac.jp
}

\begin{abstract}
We have developed prototype resistive plate chambers (RPCs) for the LEPS2 experiment at SPring-8 in Japan. At LEPS2, RPCs will be used as time-of-flight detectors. The goal is to achieve a time resolution of $50 \mathrm{ps}$ with a readout cell as large as possible. We made several types of RPCs and examined the dependence of time resolution with the size and the shape of the readout cell. We found that the time resolution and the efficiency largely depend on the shape of the readout cell and the position where it is excited. We confirmed that two-ends strip-shaped readout cells performed better than one-end readout cells, and a promising time resolution of $60 \mathrm{ps}$ was obtained with a $2.5 \mathrm{~cm}$ (wide) $\times 108 \mathrm{~cm}$ (long) readout.
\end{abstract}

KEYWORDS: Timing detectors; Resistive-plate chambers; Instrumentation and methods for timeof-flight (TOF) spectroscopy

\footnotetext{
${ }^{1}$ Corresponding author.
} 


\section{Contents}

1 Introduction 1

2 Description of the RPCs 2

3 Experimental setup $\quad 2$

4 Test results 3

4.1 General behavior 3

4.2 Pads 5

4.2.1 Dependence with size 5

4.2.2 Dependence with the position of the readout line 5

$\begin{array}{lll}4.2 .3 & \text { Position dependence } & 6\end{array}$

$\begin{array}{lll}4.3 & \text { Strips } & 6\end{array}$

$\begin{array}{lll}\text { 4.3.1 Dependence with width and length } & 7\end{array}$

4.3.2 Position dependence $\quad 8$

5 Conclusion 9

\section{Introduction}

The construction of LEPS2 (Laser Electron Photon experiment at SPring-8), a new beamline for hadron photo-production experiments, has started at SPring-8 in Japan. The photon beam is produced by backward Compton scattering (BCS) of laser photons with $8 \mathrm{GeV}$ electrons circulating in SPring-8 storage ring. Energy range of photon beam is $1.5-3.0 \mathrm{GeV}$. Hadrons with strange quarks are produced by photo-production. The LEPS experiment has reported various interesting data whereas the detection area was limited to forward angle $[1,2]$. The new LEPS2 detectors will cover almost $4 \pi$ solid angle. Moreover, the beam intensity will be $10^{7} / \mathrm{s}$, which is one order of magnitude larger than LEPS.

In figure 1, a schematic drawing of LEPS2 detectors is shown. The solenoid magnet was moved from Brookhaven National Laboratory in the U.S., which was used for the AGS E787/E949 experiment. Time-of-flight (TOF) counters will cover a barrel region of $10 \mathrm{~m}^{2}$ inside the magnet. The radius of the barrel is $0.9 \mathrm{~m}$ and the depth is $2.0 \mathrm{~m}$. The flight length from the target is $0.9-$ $1.7 \mathrm{~m}$. In order to separate kaons from pions up to $1.1 \mathrm{GeV} / \mathrm{c}$ via a TOF measurement, $50 \mathrm{ps}$ TOF time resolution is required. We decided to use resistive plate chambers (RPCs) for our TOF system. A RPC is a gas counter made of resistive plates. It is proved that multi thin gap RPCs can achieve an excellent time resolution $[3,4]$ and RPCs are now used as TOF counters in many experiments [5, 6].

In addition to the resolution, the number of channels is an important requirement of our RPCs. In order to reduce the cost of the readout circuit, we should reduce the number of channels as much 

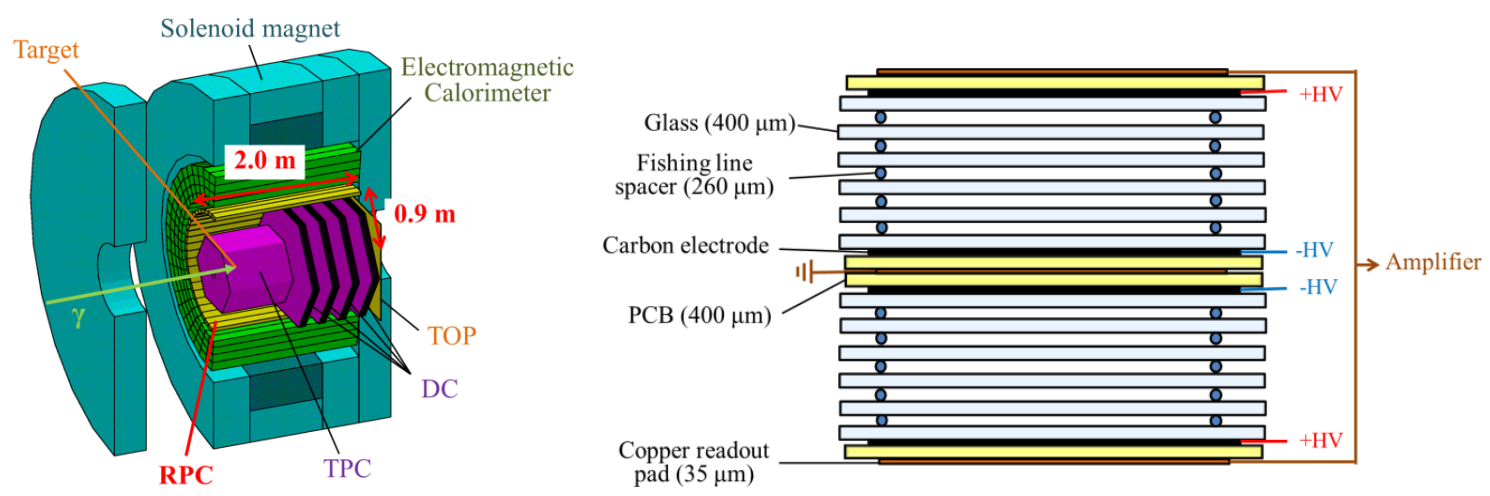

Figure 1. Schematic drawing of the LEPS2 detector. RPCs cover the barrel region (painted yellow). The radius of the

Figure 2. Schematic drawing of a prototype RPC. The anode readout is connected to the amplifier and the cathode readout is connected to ground.

barrel is $0.9 \mathrm{~m}$ and the depth is $2.0 \mathrm{~m}$.

as possible. A realistic goal is a number of channels less than 2000. It means a cell read by one channel has to be larger than $50 \mathrm{~cm}^{2}$. An efficiency better than $99 \%$ is also required because signals of RPCs will be used as trigger for DAQ. Since the particles which arrive at the barrel are mostly hadrons and the production rate of hadrons stemming from gamma-ray interactions is quite low, high rate capability is not required for our RPCs and a rate capability up to $1 \mathrm{~Hz} / \mathrm{cm}^{2}$ is sufficient.

\section{Description of the RPCs}

We developed various types of RPCs with different gap size, number of stacks and readout cell geometries. The gap size was defined by the diameter of the fishing line used as a spacer and was $148 \mu \mathrm{m}$ and $260 \mu \mathrm{m}$. The number of stacks was one, two and four, and the number of gaps in a single stack was 6 for $148 \mu \mathrm{m}$ and 5 for $260 \mu \mathrm{m}$ gap RPC. We developed two different size RPCs. The first one consisted of glasses with an area of $10 \mathrm{~cm} \times 50 \mathrm{~cm}$ (configuration (A)) and the other was $15 \mathrm{~cm} \times 110 \mathrm{~cm}$ (configuration (B)). The thickness of the glass was $400 \mu \mathrm{m}$ for both configurations. A schematic drawing of the cross section of our typical RPC is shown in figure 2. Double-sided adhesive carbon tapes ${ }^{1}$ were used as electrodes. The surface resistivity of carbon tape was $500 \Omega / \square$ for configuration (A) and $10 \mathrm{M} \Omega / \square$ for configuration (B). A copper tape was used for the $20 \mathrm{~cm}$ and $40 \mathrm{~cm}$ long strip readout while copper paint was used for the other readout cells. The thickness of copper of both tapes and paint was $35 \mu \mathrm{m}$. Anode readout was connected to the amplifiers and cathode readout was connected to ground. The gas mixture was $90 \% \mathrm{C}_{2} \mathrm{H}_{2} \mathrm{~F}_{4}$, $5 \% \mathrm{SF}_{6}$ and $5 \%$ iso- $\mathrm{C}_{4} \mathrm{H}_{10}$.

\section{Experimental setup}

Beam test was performed at LEPS beamline. The experimental setup is shown in figure 3 . The BCS gamma-rays were injected to a lead converter and electrons and positrons were produced via pair-creation. The electrons with energy around $1.5 \mathrm{GeV}$ were bent with a dipole magnet and

\footnotetext{
${ }^{1}$ T-9145 and T-9188 Carbon tape, ESD EMI Engineering Corporation, Tokyo, Japan.
} 


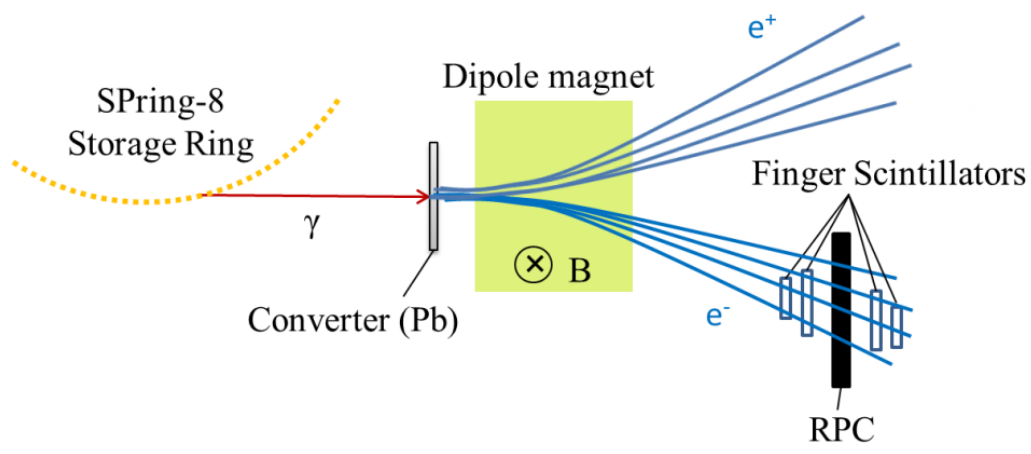

Figure 3. Experimental setup of beam test at LEPS. The gamma rays are converted to electron- positron pairs. Electrons are bent with a solenoid magnet and hit the RPC.

passed through the RPC. The trigger area was defined with four finger scintillators as $1 \times 2 \mathrm{~cm}^{2}$. We kept the trigger rate as $5-20 \mathrm{~Hz} / \mathrm{cm}^{2}$, since it satisfied the requirement of LEPS2. We used a NIM amplifier, KN2104 manufactured by Kaizu Works, for signal amplification. The input impedance of the amplifier was $50 \Omega$ and the gain was about 5 at $500 \mathrm{MHz}$. Because the gain was not enough, we used the amplifier three times in series. The data acquisition system was based on CAMAC. The TOF of electrons from the converter to RPC was measured with a high resolution TDC module with 25 ps time bin, GNC-040 made by DNomes Design. The time resolution of the TDC was about 18 ps. The charge information was obtained with a Repic RPC-022 ADC and slewing correction was applied to the time measurement. At LEPS and LEPS2, the RF signal is used as the start timing of TOF measurement [7]. The RF signal is provided at the timing of the center of electron bunch in the SPring-8 storage ring. The width of the electron bunch is $14-18 \mathrm{ps}$ depending on the filling pattern of the electrons in the storage ring. Since both electrons in the storage ring and BCS photons fly at the same (light) speed, the time distribution of electrons is conserved after BCS. Therefore, the ambiguity of the timing of the pair creation at the converter is the time difference between the RF (center of bunch) and the electron for which BCS was occurred. This time difference corresponds to the width of the electron bunch; 14-18 ps. Thus, we can decide the start timing with the resolution below 20 ps by using RF signals.

\section{Test results}

Here we show the results of the beam test. We started from small readout cell $(1.5 \mathrm{~cm} \times 5.5 \mathrm{~cm})$ and examined the gap configuration effect on the time resolution and the efficiency. Then we tested different readout cell geometries. In this article, a "pad" refers to a readout cell which signals are read from one end and a "strip" refers to a readout cell which signals are read from two ends. The time resolution reported here includes the start jitter from the RF signal, front-end electronics and the RPC.

\subsection{General behavior}

A typical time vs. charge scatter plot is shown in figure 4. After a time-slewing correction by 6 order polynomial, the time resolution is obtained by the standard deviation of a Gaussian fit 

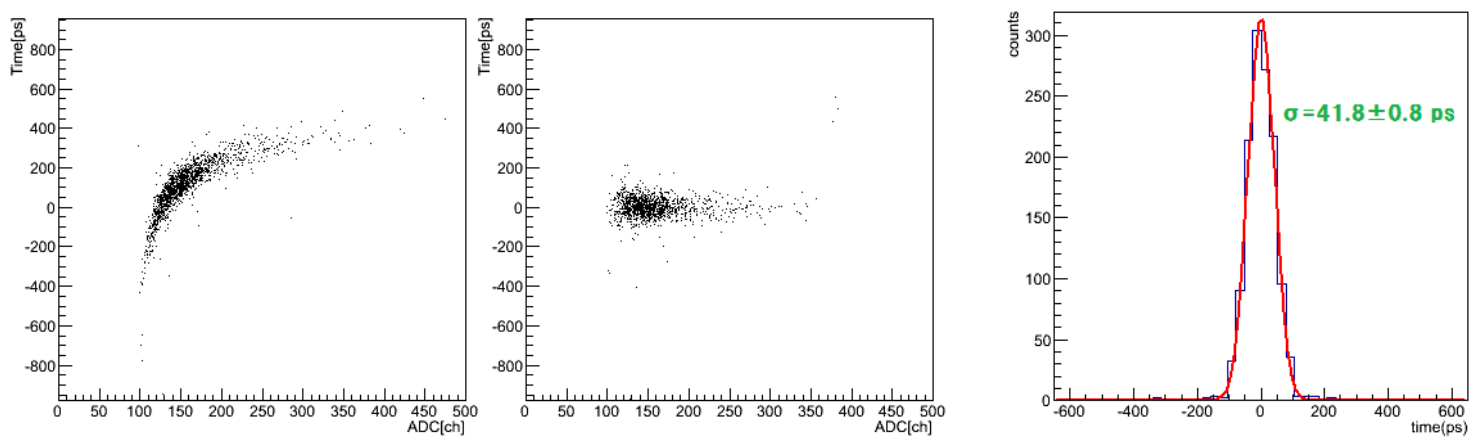

Figure 4. A time vs. charge scatter plot. Before time-slewing correction (left) and after correction (right). This is the plot of the $148 \mu \mathrm{m}$ gap four-stack RPC with an applied voltage of $11.5 \mathrm{kV}$.

Figure 5. A timing distribution after slewing correction. This figure corresponds to the projection of time axis of figure 4 (right).

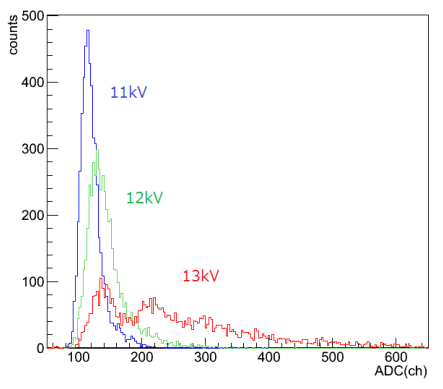

Figure 6. A charge distribution at different applied voltage. This is the plot of the $148 \mu \mathrm{m}$ gap double-stack RPC. At $13 \mathrm{kV}$, streamers appear and there are two peaks corresponding to avalanches and streamers, respectively.

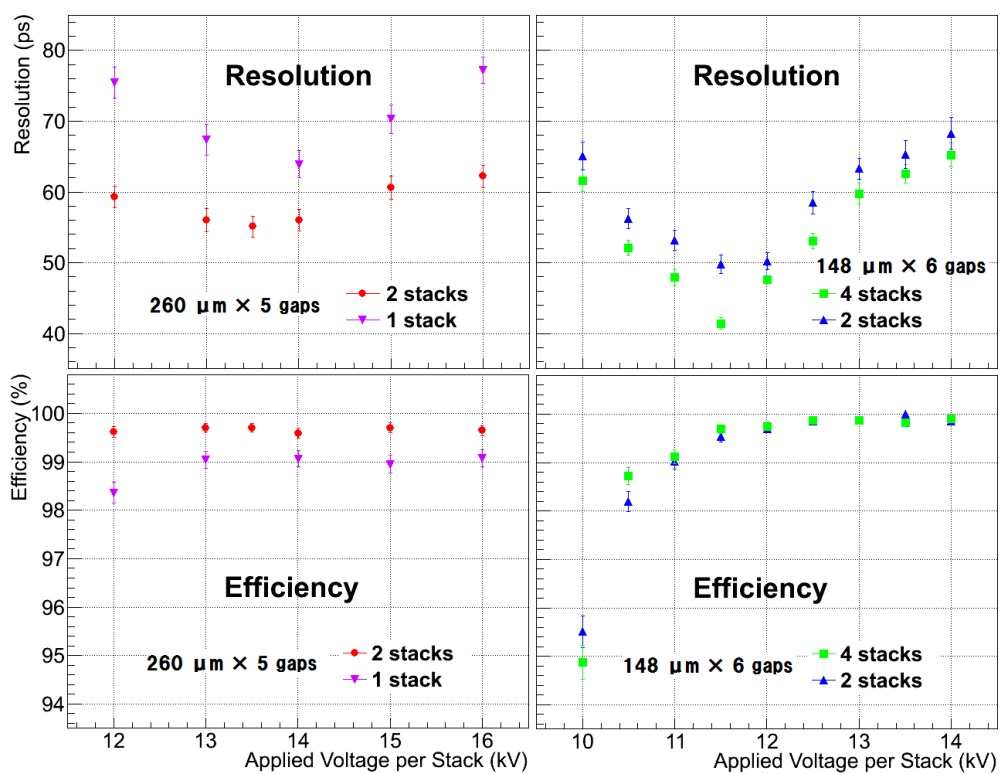

Figure 7. The time resolution and the efficiency of the $260 \mu \mathrm{m}$ (left) and the $148 \mu \mathrm{m}$ gap RPC (right) in different stack configuration. The time resolution improved when the number of stacks is increased.

(figure 5). In figure 6, the charge distribution at different applied voltage is shown. Above a certain value of voltage, streamers appeared and time-slewing corrections did not work correctly.

The time resolution and the efficiency of different gap configurations are shown in figure 7 . The readout pad was $1.5 \mathrm{~cm}$ (wide) $\times 5.5 \mathrm{~cm}$ (long) and the trigger was $1 \mathrm{~cm}$ (wide) $\times 2 \mathrm{~cm}$ (long). With double-stack configuration, both $148 \mu \mathrm{m}$-wide and $260 \mu \mathrm{m}$-wide gap achieved a time resolution around $50 \mathrm{ps}$ and an efficiency better than $99 \%$. There was no big difference between $148 \mu \mathrm{m}$ and $260 \mu \mathrm{m}$ and we confirmed that double-stack RPC has an ability to achieve the time resolution and the efficiency required at LEPS2. We also tested one-stack RPC with $260 \mu \mathrm{m}$-wide 
Table 1. The time resolution and the efficiency for different sizes of the readout pad. Both the time resolution and the efficiency become worse with increasing pad size.

\begin{tabular}{|c|c|c|c|}
\hline Readout pad & $\begin{array}{c}\text { Number of channels } \\
\text { at LEPS2 }\end{array}$ & Resolution & Efficiency \\
\hline $1.5 \mathrm{~cm} \times 5.5 \mathrm{~cm}$ & 14,000 & $50 \pm 1 \mathrm{ps}$ & $99.5 \pm 0.1 \%$ \\
\hline $2.5 \mathrm{~cm} \times 3.7 \mathrm{~cm}$ & 13,000 & $57 \pm 1 \mathrm{ps}$ & $98.8 \pm 0.2 \%$ \\
\hline $2.5 \mathrm{~cm} \times 7.4 \mathrm{~cm}$ & 6,000 & $65 \pm 3 \mathrm{ps}$ & $99.4 \pm 0.2 \%$ \\
\hline $5.0 \mathrm{~cm} \times 7.4 \mathrm{~cm}$ & 3,000 & $85 \pm 3 \mathrm{ps}$ & $97.3 \pm 0.3 \%$ \\
\hline $10.0 \mathrm{~cm} \times 7.4 \mathrm{~cm}$ & 1,600 & $151 \pm 3 \mathrm{ps}$ & $90.0 \pm 0.5 \%$ \\
\hline
\end{tabular}

gap and four-stack RPC with $148 \mu \mathrm{m}$-wide gap. By changing from one-stack to double-stack, the time resolution improved about $10 \mathrm{ps}$ and the efficiency increased about $1 \%$. On the other hand, from double-stack to four-stack, the time resolution improved about $5 \mathrm{ps}$ but the efficiency did not improve because the double-stack RPC already achieved an efficiency better than $99 \%$. The fourstack RPC achieved the best time resolution of $40 \mathrm{ps}$. We confirmed that the number of gaps has a large effect on the time resolution.

\subsection{Pads}

\subsubsection{Dependence with size}

We tested five different size pads on a double-stack $148 \mu \mathrm{m}$ gap RPC. The smallest one was $1.5 \mathrm{~cm}$ (wide) $\times 5.5 \mathrm{~cm}$ (long), corresponding to 14,000 channels if they were used at LEPS 2 experiment and the largest was $10 \mathrm{~cm}$ (wide) $\times 7.4 \mathrm{~cm}$ (long), corresponding to 1,600 channels. The trigger was at the center of the pad and the area was $1 \mathrm{~cm}$ (wide) $\times 2 \mathrm{~cm}$ (long). The applied voltage across one stack (6 gaps) was $12 \mathrm{kV}$. Table 1 shows the resolution and the efficiency measured with each readout pad. Both the resolution and the efficiency become worse with increasing the pad size. It is thought that the performance of large pads is worse because their signal is composed of many signals with different pathway. In addition, this effect can be also interpreted as a larger capacitive behavior as shown in [8]. Both descriptions are largely equivalent for electrically short cells.

\subsubsection{Dependence with the position of the readout line}

We tested $2.5 \mathrm{~cm}$ (wide) $\times 7.4 \mathrm{~cm}$ (long) pad with different position and different shape of the readout line. The trigger was at the center of the pad and the area was $1 \mathrm{~cm}$ (wide) $\times 2 \mathrm{~cm}$ (long). The shapes (A-E) are shown in figure 8 and the results are shown in table 2. Comparing A and B, one can find that the time resolution is better when the readout line locates at the center. The shape of readout line did not affect to the performance (A and C). On the other hand, comparing A, C and $\mathrm{D}, \mathrm{E}$, one can find that the resolution and the efficiency become worse when a readout line locates at longer side. Since five structures have approximately the same capacitance, we attribute the effect to propagation phenomena due to different path-ways in each case, reminiscent of a transmission line behavior [8]. We confirmed that not only the pad size but also the readout line position affects to the performance of RPC. 


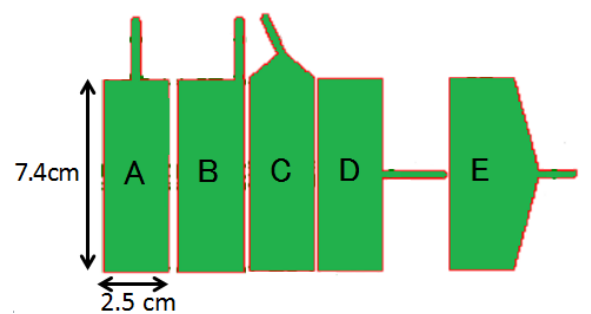

Figure 8. Similar pad geometries with different positions of the readout line. The pad size was $2.5 \mathrm{~cm} \times 7.4 \mathrm{~cm}$.

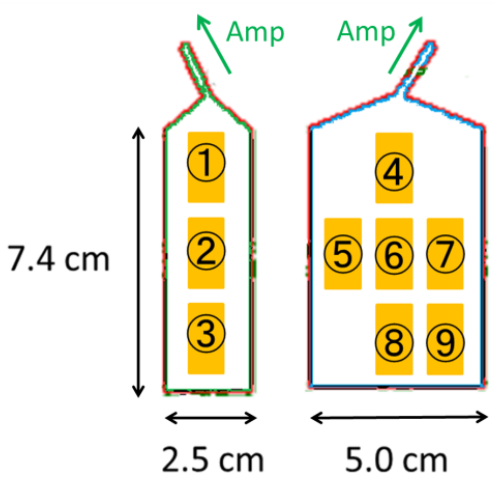

Figure 9. Various positions studied over two different pad geometries. The pad areas were $2.5 \mathrm{~cm} \times 7.4 \mathrm{~cm}$ and $5.0 \mathrm{~cm} \times$ $7.4 \mathrm{~cm}$. The trigger area was $1 \mathrm{~cm} \times 2 \mathrm{~cm}$.
Table 2. The time resolution and the efficiency for different positions of the readout line.

\begin{tabular}{|c|c|c|}
\hline Readout pad & Resolution & Efficiency \\
\hline A & $65 \pm 3 \mathrm{ps}$ & $99.4 \pm 0.2 \%$ \\
\hline B & $74 \pm 2 \mathrm{ps}$ & $99.0 \pm 0.2 \%$ \\
\hline C & $65 \pm 2 \mathrm{ps}$ & $99.6 \pm 0.1 \%$ \\
\hline D & $78 \pm 2 \mathrm{ps}$ & $96.5 \pm 0.3 \%$ \\
\hline E & $84 \pm 2 \mathrm{ps}$ & $98.0 \pm 0.2 \%$ \\
\hline
\end{tabular}

Table 3. The position dependence of the time resolution and the efficiency of two pads. The trigger position is shown in figure 4 . The resolution became worse near the read out line.

\begin{tabular}{|c|c|c|c|}
\hline Pad & Position & Resolution & Efficiency \\
\hline \multirow{3}{*}{$2.5 \mathrm{~cm} \times 7.4 \mathrm{~cm}$} & 1 & $72 \pm 2 \mathrm{ps}$ & $99.4 \pm 0.1 \%$ \\
\cline { 2 - 4 } & 2 & $63 \pm 1 \mathrm{ps}$ & $99.8 \pm 0.1 \%$ \\
\cline { 2 - 4 } & 3 & $50 \pm 3 \mathrm{ps}$ & $99.6 \pm 0.1 \%$ \\
\hline \multirow{3}{*}{$5.0 \mathrm{~cm} \times 7.4 \mathrm{~cm}$} & 4 & $90 \pm 3 \mathrm{ps}$ & $97.5 \pm 0.3 \%$ \\
\cline { 2 - 4 } & 5 & $83 \pm 2 \mathrm{ps}$ & $97.0 \pm 0.3 \%$ \\
\cline { 2 - 4 } & 6 & $78 \pm 2 \mathrm{ps}$ & $97.8 \pm 0.2 \%$ \\
\cline { 2 - 4 } & 7 & $75 \pm 2 \mathrm{ps}$ & $96.7 \pm 0.3 \%$ \\
\cline { 2 - 4 } & 9 & $67 \pm 2 \mathrm{ps}$ & $95.9 \pm 0.3 \%$ \\
\hline
\end{tabular}

\subsubsection{Position dependence}

We examined the position dependence of the time resolution and the efficiency by moving the position of the trigger scintillators. The trigger position and the results of two pads with the size of $2.5 \mathrm{~cm} \times 7.4 \mathrm{~cm}$ and $5.0 \mathrm{~cm} \times 7.4 \mathrm{~cm}$ are shown in figure 9 and table 3 . One can compare the results of position 1,2,3 and 4,6,8 and find that the time resolution became worse near the readout line. It is thought that the resolution became worse near the readout line because the direct signal is superimposed on the reflected signal at the other end, again suggestive of transmission line like behavior. There is no position dependence on the efficiency.

We found that it is difficult to achieve a good resolution with large, one-end readout pad so we developed double-ended strip-readout.

\subsection{Strips}

We developed three different sizes of strip-readouts on $148 \mu \mathrm{m}$ and $260 \mu \mathrm{m}$ gap double-stack RPCs in configuration (A). The size of the strip was $2.5 \mathrm{~cm}$ (wide) $\times 20 \mathrm{~cm}$ (long), $1.5 \mathrm{~cm}$ (wide) $\times 40 \mathrm{~cm}$ (long) and $2.5 \mathrm{~cm}$ (wide) $\times 40 \mathrm{~cm}$ (long). The longer strip is developed in configuration (B). The size of strip was $2.5 \mathrm{~cm}$ (wide) $\times 108 \mathrm{~cm}$ (long) and the gap was $260 \mu \mathrm{m}$ double-stack configuration. We measured signals from both ends and averaged the timing. The trigger area was $1 \mathrm{~cm}$ (wide) $\times$ 


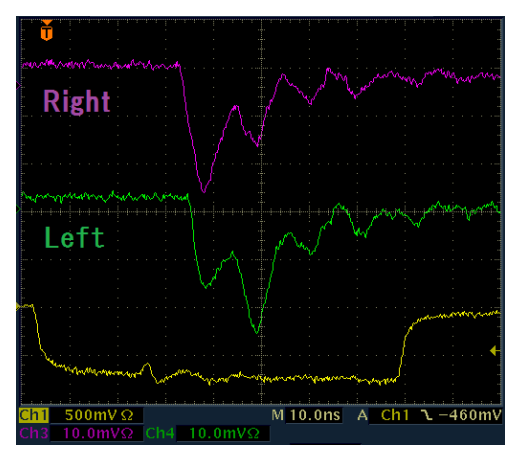

(a)

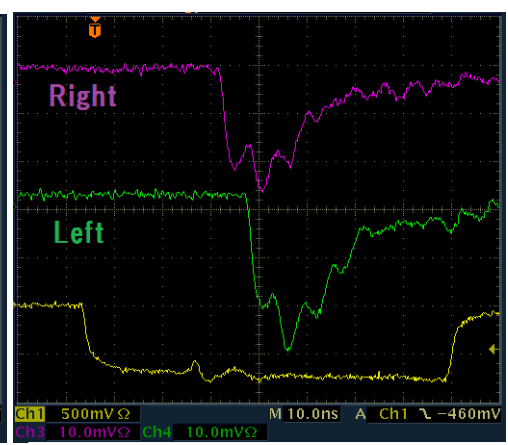

(b)

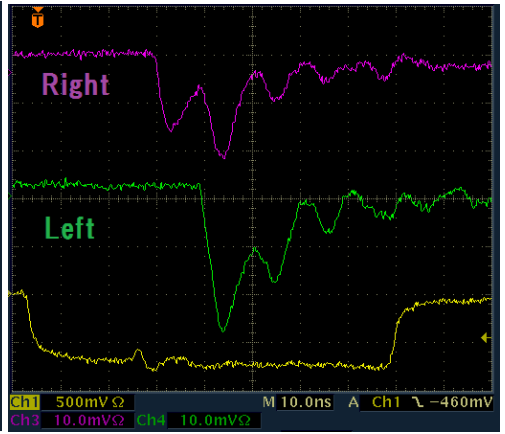

(c)

Figure 10. Signal shape of $2.5 \mathrm{~cm} \times 108 \mathrm{~cm}$ strip. The trigger position is $1 \mathrm{~cm}$ from the left-end (a), center (b) and $24 \mathrm{~cm}$ from the right-end (c). The upper (purple) signal is the signal of right-end, the center (green) is left-end and the lower (yellow) is the trigger signal.

Table 4. The time resolution and the efficiency of several strips. There was no big difference regarding the time resolution for all geometries. The efficiency was distinctly lower for the $148 \mu \mathrm{m}$ gap size.

\begin{tabular}{|c|c|c|c|c|}
\hline Gap width & Readout strip & $\begin{array}{c}\text { Channel } \\
\text { at LEPS2 }\end{array}$ & Resolution & Efficiency \\
\hline \multirow{3}{*}{$148 \mu \mathrm{m}$} & $2.5 \mathrm{~cm} \times 20 \mathrm{~cm}$ & 4,000 & $61 \pm 3 \mathrm{ps}$ & $92.8 \pm 0.4 \%$ \\
\cline { 2 - 5 } & $1.5 \mathrm{~cm} \times 40 \mathrm{~cm}$ & 3,400 & $62 \pm 2 \mathrm{ps}$ & $96.4 \pm 0.3 \%$ \\
\cline { 2 - 5 } & $2.5 \mathrm{~cm} \times 40 \mathrm{~cm}$ & 2,000 & $63 \pm 2 \mathrm{ps}$ & $90.4 \pm 0.5 \%$ \\
\hline \multirow{4}{*}{$260 \mu \mathrm{m}$} & $2.5 \mathrm{~cm} \times 20 \mathrm{~cm}$ & 4,000 & $56 \pm 2 \mathrm{ps}$ & $99.2 \pm 0.2 \%$ \\
\cline { 2 - 5 } & $1.5 \mathrm{~cm} \times 40 \mathrm{~cm}$ & 3,400 & $64 \pm 2 \mathrm{ps}$ & $98.4 \pm 0.3 \%$ \\
\cline { 2 - 5 } & $2.5 \mathrm{~cm} \times 40 \mathrm{~cm}$ & 2,000 & $61 \pm 2 \mathrm{ps}$ & $98.6 \pm 0.2 \%$ \\
\cline { 2 - 5 } & $2.5 \mathrm{~cm} \times 108 \mathrm{~cm}$ & 800 & $58 \pm 2 \mathrm{ps}$ & $99.7 \pm 0.1 \%$ \\
\hline
\end{tabular}

$2 \mathrm{~cm}$ (long). Figure 10 shows the signal shape of $2.5 \mathrm{~cm} \times 108 \mathrm{~cm}$ strip. Figure 10(a) shows signals at $1 \mathrm{~cm}$ from left-end, 10(b) is at the center and 10 (c) is $24 \mathrm{~cm}$ from right-end. The signal of left side is delayed by $5 \mathrm{~ns}$ from the right side since the cable of left side is about $1 \mathrm{~m}$ longer than right side. Because of impedance mismatch between the strip and the amplifier, reflections occur. The impedance of the strip can be calculated as follows: the pulse height of the first pulse $\left(\mathrm{T}_{1}\right)$ and the second pulse $\left(\mathrm{T}_{2}\right)$ of left signal of figure $10(\mathrm{a})$ have a relation with reflection coefficient $(\mathrm{R})$ : $\mathrm{T}_{2} / \mathrm{T}_{1}=\mathrm{R}+\mathrm{R}^{2}$. The reflection coefficient $\mathrm{R}$ can be written as a function of the impedance of strip $\left(Z_{\mathrm{s}}\right)$ and the impedance of the circuit $\left(\mathrm{Z}_{\mathrm{c}}\right): \mathrm{R}=\left(\mathrm{Z}_{\mathrm{c}}-\mathrm{Z}_{\mathrm{s}}\right) /\left(\mathrm{Z}_{\mathrm{c}}+\mathrm{Z}_{\mathrm{s}}\right)$. By substituting $\mathrm{T}_{1}=20 \mathrm{mV}, \mathrm{T}_{2}=$ $30 \mathrm{mV}$ and $Z_{\mathrm{c}}=50 \Omega$, one can extract $\mathrm{Z}_{\mathrm{s}} \sim 5 \Omega$.

\subsubsection{Dependence with width and length}

Table 4 shows the time resolution and the efficiency of each strip. During the measurement, the trigger position was set to be at the center of the strip. The applied voltage was $12 \mathrm{kV}(148 \mu \mathrm{m}$ gap) and $14 \mathrm{kV}(260 \mu \mathrm{m}$ gap). The time resolutions and the efficiencies of the $260 \mu \mathrm{m}$ gap RPC were almost the same value for all shapes and they did not depend on the strip length. The $260 \mu \mathrm{m}$ gap RPCs achieved close to $99 \%$ efficiency but those of $148 \mu \mathrm{m}$ gap RPC were 90 to $97 \%$. The $2.5 \mathrm{~cm}$ 


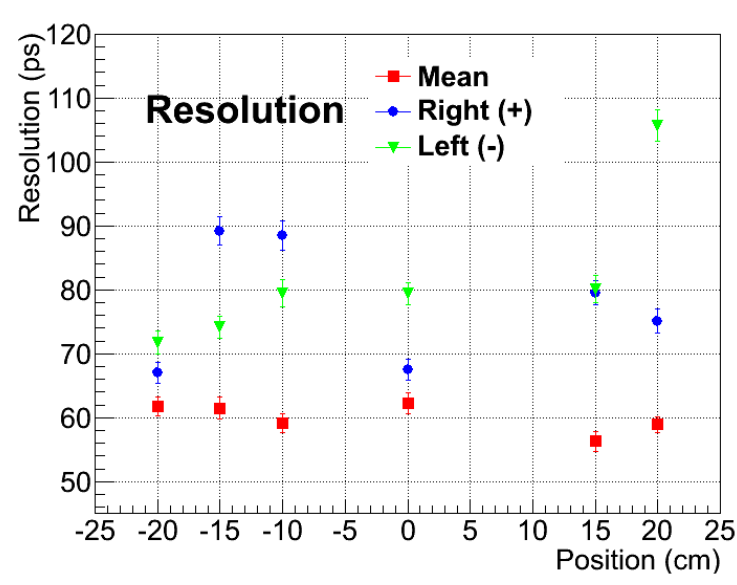

Figure 11. The time resolution as a function of the position along $2.5 \mathrm{~cm} \times 40 \mathrm{~cm}$ strip readout. The resolution of the mean time showed no position dependence, contrary to the one obtained for each end separately.

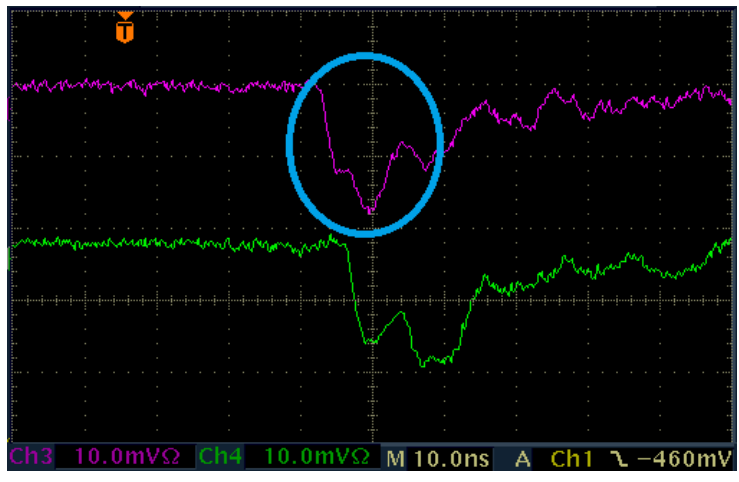

Figure 12. Signals of strip readout. At some positions, the first peak was the superimposition of two pulses like of the upper (purple) signal (circled). The time- slewing correction did not work well with such a signal and the time resolution was worse than at other positions.

$\times 108 \mathrm{~cm}$ readout strip, corresponding to 800 channels at LEPS2, achieved $60 \mathrm{ps}$ time resolution and $99 \%$ efficiency. Although the time resolution is slightly worse than the requirement of LEPS2, we confirmed that we can significantly reduce the number of channels keeping good resolution and efficiency by employing strip-shaped readout cells with $260 \mu \mathrm{m}$ gap RPC.

\subsubsection{Position dependence}

The time resolution at different trigger positions on $2.5 \mathrm{~cm} \times 40 \mathrm{~cm}$ readout strip is shown in figure 11. Although there was no big position dependence on the mean resolution, a large position dependence was observed in each end. The resolution of each end was worse at the position where the slewing correction did not work sufficiently well. At these positions, the first peak was superimposed with two pulses like shown in figure 12 and the charge information did not correspond correctly with the slewing rate of the first peak. The shape of signal strongly depended on the position and changed drastically with a few mm difference of the trigger position, due to the impedance mismatch between our readout chain and the strip. We think that the signal shape is sensitive to small variations of the trigger position because the propagation path and the propagation time of the signal vary with the small change of the position relation between the trigger and the readout line. The resolution of each end shown in figure 11 was asymmetric because the accuracy of our trigger alignment was a few $\mathrm{mm}$ and the trigger was not exactly at the same position on the right and the left side. Although the signal shape of one-end was not suited for the time-slewing correction at some positions, the shape of the other end was fine. The deformation of the signal of only one end did not affect to the mean resolution. Hence, the 60 ps time resolution was achieved everywhere on the strip. 


\section{Conclusion}

We examined the dependence of RPC performance with the gap configuration and the readout cell geometry. The time resolution was improved with increasing the number of stacks and the efficiency was better with $260 \mu \mathrm{m}$ gap than $148 \mu \mathrm{m}$ gap. We found that it is difficult to achieve good time resolutions with large one-end readout pads. Thereafter, we confirmed that double-end stripshaped readout cells have a good time resolution which is uniform along the strip. Furthermore, the time resolution does not depend on the strip length in the range of $20 \mathrm{~cm}$ to $100 \mathrm{~cm}$.

The RPC with $2.5 \mathrm{~cm} \times 108 \mathrm{~cm}$ readout strip achieved $60 \mathrm{ps}$ TOF resolution and an efficiency better than $99 \%$. This readout cell size corresponds to 800 channels at LEPS2 and these results are very close to LEPS2 requirements. The time resolution includes the jitter of the circuits and it is estimated to be about $40 \mathrm{ps}$ [9]. Thus, it is important to develop low-jitter amplifiers and TDCs to improve the time resolution further.

\section{Acknowledgments}

This work is supported by the Grant-in-Aid for scientific research of MEXT/JSPS (No. 24105711) and the Grant-in-Aid for JSPS Fellow (No. 24608).

\section{References}

[1] LEPS collaboration, T. Mibe et al., Diffractive phi-meson photoproduction on proton near threshold, Phys. Rev. Lett. 95 (2005) 182001 [nucl-ex/0506015].

[2] LEPS collaboration, T. Nakano et al., Evidence of the $\Theta^{+}$in the $\gamma d \rightarrow K^{+} K^{-}$pn reaction, Phys. Rev. C 79 (2009) 025210 [arXiv : 0812.1035].

[3] E. Cerron Zeballos, I. Crotty, D. Hatzifotiadou, J. Lamas Valverde, S. Neupane, et al., A New type of resistive plate chamber: The Multigap RPC, Nucl. Instrum. Meth. A 374 (1996) 132.

[4] ALICE collaboration, P. Fonte, A. Smirnitsky and M. Williams, A New high resolution TOF technology, Nucl. Instrum. Meth. A 443 (2000) 201.

[5] A. Akindinov, A. Alici, F. Anselmo, P. Antonioli, M. Basile, et al., Latest results on the performance of the multigap resistive plate chamber used for the ALICE TOF, Nucl. Instrum. Meth. A 533 (2004) 74 [INSPIRE].

[6] Y. Wang, Y.-J. Li, J.-P. Cheng, J. Li, Q. Yue, et al., Study on the performance of multi-gap resistive plate chambers, Nucl. Instrum. Meth. A 538 (2005) 425.

[7] T. Nakano, J. Ahn, M. Fujiwara, H. Kohri, N. Matsuoka, et al., Multi-GeV laser electron photon project at SPring-8, Nucl. Phys. A 684 (2001) 71.

[8] D. Gonzalez-Diaz et al., RPC simulations from a current stand point, in proceedings of XI workshop on Resistive Plate Chambers and Related Detectors (RPC2012), February, 5-10, 2012, INFN, Italy [PoS (RPC2012) 035].

[9] N. Tomida et al., Development of large readout area, high time resolution RPCs for LEPS2 at SPring-8, in proceedings of XI workshop on Resistive Plate Chambers and Related Detectors (RPC2012), February, 5-10, 2012, INFN, Italy [PoS (RPC2012) 017]. 